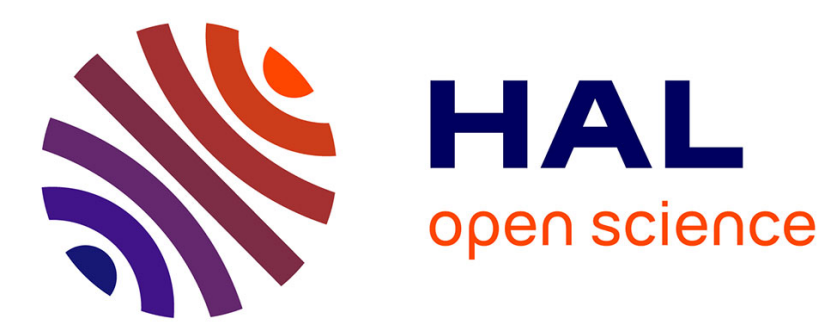

\title{
Experimental study of the elastic properties of a triangular percolation network
}

\author{
A. Gilabert, M. Ben-Ayad, S. Roux, E. Guyon
}

\section{To cite this version:}

A. Gilabert, M. Ben-Ayad, S. Roux, E. Guyon. Experimental study of the elastic properties of a triangular percolation network. Journal de Physique, 1988, 49 (10), pp.1629-1634. 10.1051/jphys:0198800490100162900 . jpa-00210843

\section{HAL Id: jpa-00210843 https://hal.science/jpa-00210843}

Submitted on 1 Jan 1988

HAL is a multi-disciplinary open access archive for the deposit and dissemination of scientific research documents, whether they are published or not. The documents may come from teaching and research institutions in France or abroad, or from public or private research centers.
L'archive ouverte pluridisciplinaire HAL, est destinée au dépôt et à la diffusion de documents scientifiques de niveau recherche, publiés ou non, émanant des établissements d'enseignement et de recherche français ou étrangers, des laboratoires publics ou privés. 


\title{
LE JOURNAL DE PHYSIQUE
}

J. Phys. France 49 (1988) 1629-1634

OCTOBRE 1988, PAGE 1629

Classification

Physics Abstracts

$62.20-64.40 \mathrm{~A}$

\section{Short communication}

\section{Experimental study of the elastic properties of a triangular per- colation network}

\author{
A. Gilabert $\left({ }^{1}\right)$, M. Ben-Ayad $\left({ }^{1}\right)$, S. $\operatorname{Roux}\left({ }^{2}\right)$ and E. Guyon $\left({ }^{2}\right)$
}

( ${ }^{1}$ Laboratoire de Physique de la Matière Condensée, UA 190, Université de Nice, Parc Valrose, 06034 Nice Cedex, France

$\left({ }^{2}\right)$ Laboratoire d'Hydrodynamique et Mécanique Physique, UA 857, E.S.P.C.I., 10 rue Vauquelin, 75231 Paris Cedex, France

(Reçu le 22 juin 1988, accepté le 27 juillet 1988)

Résumé.- Nous étudions expérimentalement les propriétés élastiques d'un réseau triangulaire de barres élastiques réticulées, dont une proportion $q$ choisie aléatoirement est manquante. Pour $0<$ $q<q_{\mathrm{r}}=0.33 \pm 0.04$, la décroissance du module élastique peut être bien décrite par l'approximation auto-cohérente (le comportement critique est hors de portée expérimentalement). Entre $q_{\mathrm{r}}$ et le seuil de percolation, $q_{\mathrm{c}}=0.66 \pm 0.04$, le réseau peut être étiré librement avant qu'il n'acquiert une rigidité. L'évolution de cette extension libre en fonction de $q$ est étudiée. Certains comportements critiques sont proposés.

\footnotetext{
Abstract.- We report on the experimental study of the elastic properties of a triangular lattice consisting of bonds free tó rotate around their junctions, and where a fraction $q$ of bonds are removed at random. For $0<q<q_{\mathrm{r}}=0.33 \pm 0.04$, the elastic-modulus decrease can be very well described by a self-consistent approximation. (The critical behavior is out of reach experimentally). Between $q_{\mathrm{r}}$ and the percolation threshold, $q_{c}=0.66 \pm 0.04$, the lattice can be stretched freely before it acquires a non-zero elastic modulus. The evolution of the free extension as a function of $q$ is studied. Some estimates of critical behaviors are proposed.
} 
The percolation problem of the elasticity of randomly depleted media has motivated a large theoretical, numerical and experimental effort in the past. Two classes of models are to be distinguished :

1) Systems with angular elasticity, where the rigidity goes to zero at the usual percolation (connectivity) threshold; and

2) Systems with central-force interactions, where the rigidity threshold and the usual percolation threshold are distinct. Whereas the first case is now a well-understood subject [1-4], the second is still quite debated [5-7]. In particular, to our knowledge no experiments have been reported in the literature up to now.

Let us call $q$ the fraction of bonds removed at random in a triangular network consisting of elastic bars freely rotating at the nodes of the lattice. For $0<q<q_{\mathrm{r}}$, the lattice is rigid and its elastic modulus becomes zero for $q=q_{\mathrm{r}}$. At this point, the lattice can be deformed freely, even though it is still fully connected. Finally, the lattice becomes disconnected into small clusters for $q$ larger than $q_{\mathrm{c}}$. In the range $q_{\mathrm{r}}<q<q_{\mathrm{c}}$, a finite deformation has got to be applied in order to render the lattice rigid again. The aim of this rapid communication is to address mainly the question of the free stretching of the lattice in that interval of $q$ values.

The experimental lattice consists of an horizontal array of one thousand bonds $(15 \times 28$ lattice) made out of plexiglass bars, $3 \mathrm{~mm}$ thick, $10 \mathrm{~mm}$ wide, at both ends of which a hole is punched which can fit an axis. The distance between the two axes is $25 \mathrm{~mm}$, and the diameter of the metallic axes is $5 \mathrm{~mm}$. The bonds are free to rotate around them. One end of the lattice is fixed and the opposite side is connected to a rigid bar, onto which a dynamometer is attached. The traction imposed onto the latter bar is directly read, and the displacement is measured with a micrometer. The effect of friction in between the bonds and the axes and onto the plane the lattice was led on, was reduced by shaking the lattice before any measurement was performed. This was checked by a good reproducibility of the experiment under cycles. The force applied to the lattice was always too small to cause any buckling out of the plane.

The experiment starts with a complete triangular lattice and bonds are progressively removed randomly. At lower tension, all the contacts between the bars and the axes are not active : in this regime, the number of active contacts (and consequently the elastic modulus) increases with the applied force. This is somewhat similar to the apparent increase of the elastic modulus of a granular array under pressure. We estimate the elastic constant of the lattice when its value has stabilized (i.e. in the range $100-200 \mathrm{~N}$ ). In the immediate vicinity of the rigidity threshold, the local deformations become rapidly large resulting in an overall nonlinear relation between force and displacement. The combination of these two sources of nonlinearity makes the extent of the linear forcedisplacement characteristic smaller and smaller as $q$ tends to $q_{\mathrm{r}}$, and prevents us from obtaining any reliable data close to the rigidity threshold. Figure 1 shows the decrease of the elastic modulus $Y / Y_{0}$ (normalized by the elastic modulus $Y_{0}$ for $q=0$ ) as a function of the proportion of bonds removed.

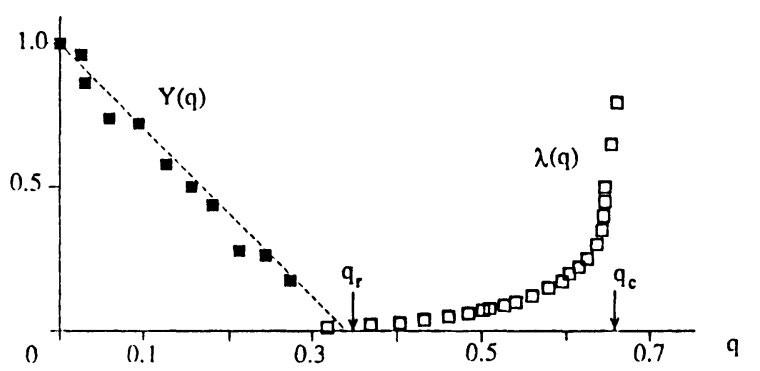

Fig. 1.- Evolution of the elastic modulus $Y(\square)$ (normalized by that of the intact lattice) and of the free extension of the lattice $\lambda(q)$ ( $\square$ in arbitrary units) as a function of the fraction, $q$, of bonds removed.

The self-consistent model prediction of the evolution of $Y / Y_{0}$ is given by [8]

$$
Y / Y_{0}=1-3 q
$$

This corresponds to the straight line on figure 1. This relation is very well verified even close to the rigidity threshold $q_{\mathrm{r}}=0.33 \pm 0.04$. This was also observed numerically in numerical simulations by Garboczi et al. [8]. The threshold $q_{\mathrm{r}}$ is also in good agreement with most previous works dealing with the critical behavior $\left(q_{\mathrm{r}}\right.$ ranging from $0.345[6]$ to $0.358[7])$.

Figure 2 shows qualitatively the evolution of the force-displacement relation as a function of $q$. In the $q-d$ plane, we have the phase diagram of the elastic state of the lattice. Above $q_{\mathrm{r}}$, there exists a domain (drawn in grey in Fig. 2) where the extension, $d$, of the lattice 
requires no force. We call $\lambda(q)$ the maximum displacement with no force applied. Obviously, $\lambda(q)$ is zero for $q \leq q_{\mathrm{r}}$ and it diverges for $q$ tending to $q_{\mathrm{c}}$. The curve $\lambda(q)$ separates two phases : one rigid, and one floppy.

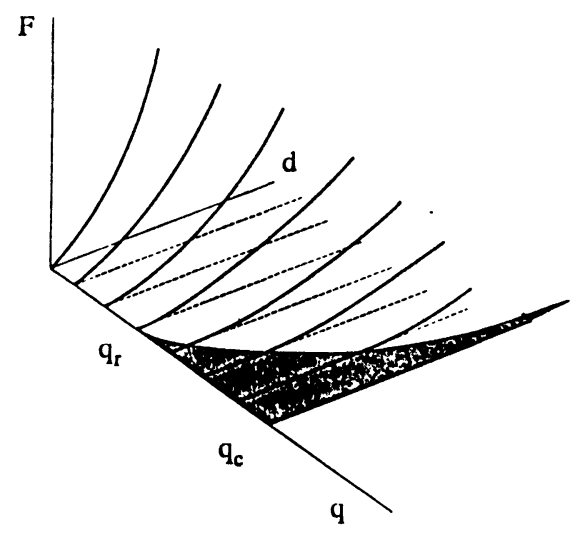

Fig. 2.- Qualitative changes in the force-displacement $(F-d)$ characteristic curve as a function of the fraction, $q$, of bonds removed. For $q$ larger than $q_{r}$, the lattice can be stretched with no force. The domain shaded in grey shows the possible displacements of the lattice with no force applied. The maximum elongation obtained is what we call the free extension, $\lambda(q)$. In particular, we study the critical behaviors of $\lambda(q)$ in the vicinity of the rigidity threshold $\left(q_{r}\right)$, and of the percolation threshold $\left(q_{\mathrm{c}}\right)$.

Figure 1 shows the evolution of $\lambda(q)$ obtained from an average over 8 lattices. The two ends of this curve are of special interest. One expects two critical behaviors :

$$
\begin{array}{ll}
\lambda(q) \propto\left(q-q_{\mathrm{r}}\right)^{a} & \text { for } q \text { close to } q_{\mathrm{r}} \\
\lambda(q) \propto\left(q_{\mathrm{c}}-q\right)^{-b} & \text { for } q \text { close to } q_{\mathrm{c}}
\end{array}
$$

From figure $3 \mathrm{a}$ and $3 \mathrm{~b}$, we can extract the slope of $\log (\lambda(q))$ versus $\log \left(q-q_{\mathrm{r}}\right)$ or $\log \left(q_{\mathrm{c}}-q\right)$, obtained with the values $q_{\mathrm{r}}=0.358$ and $q_{\mathrm{c}}=0.653$, giving approximately $a \approx 0.9 \pm$ 0.3 and $b \approx 0.3 \pm 0.2$. The theoretical values of the threshold were used since the experimental values were too unprecise to allow any determination. Once again, we want to emphasize the fact that the experimental determination of the critical behavior is a hard task to perform, and thus the exponents $a$ and $b$ should be considered as estimates more than precise values.

The divergence of $\lambda\left(q_{\mathrm{c}}\right)$ can be accounted for by the following argument : close to the usual percolation threshold, the shortest path connecting two given points is fractal, and having a fractal dimension of $d_{\min }=1.130$ [9] in two dimensions. Thus the length $\Lambda$ of the shortest path connecting two points a distance $L$ apart scales as $\Lambda \propto L^{d_{\min }}$. This property is valid if the distance $L$ is smaller than the correlation length $\xi$ which diverges at the percolation threshold as $\xi \propto\left(q_{c}-q\right)^{-\nu}$, where $\nu=4 / 3$ in two dimensions. Above the correlation length, the lattice can be seen as homogeneous, or regular. Thus, if $L$ is larger than $\xi$ the shortest path has a length of $\Lambda \propto L .\left(\xi^{d_{\min }}\right) / \xi$. (It is obviously linear in $L$ and matches the above expression for $L=\xi)$. Obviously the free extension of the lattice cannot be larger than the length of the shortest path connecting the two ends of the lattice. We thus expect $\lambda(q)$ to diverge close to $q_{c}$ with the constraint :

$$
\lambda(q)<A L \cdot\left(\xi^{d_{\min }}\right) / \xi \propto L\left(q_{\mathrm{c}}-q\right)^{-\nu\left(d_{\min }-1\right)}
$$

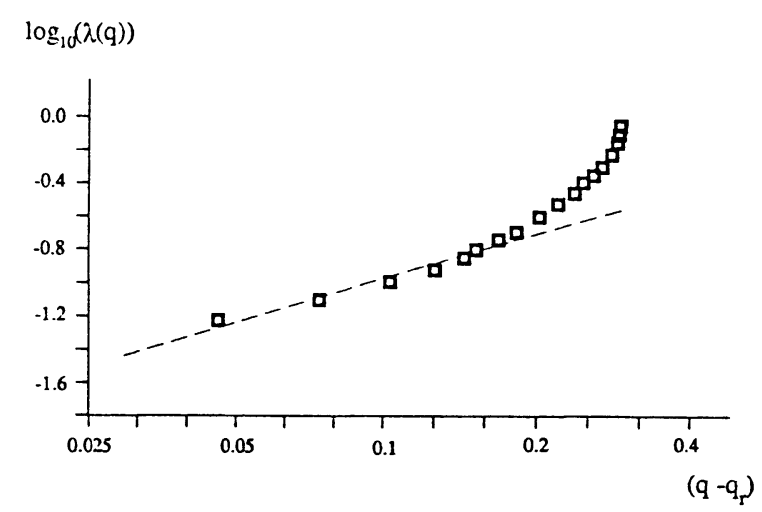

A

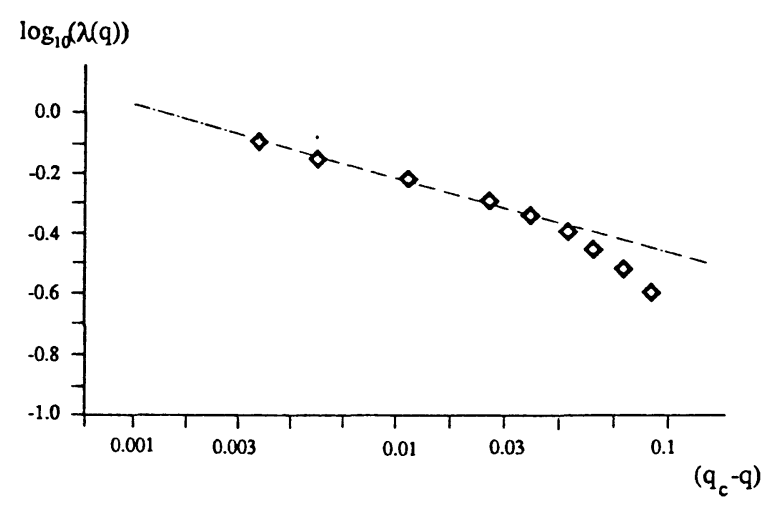

B

Fig. 3. - Log-log plot of the free extension $\lambda(q)$ as a function of $\left(q-q_{\mathrm{r}}\right)(\mathrm{A})$ and of $\left(q_{\mathrm{c}}-q\right)$ (B). The slope of this two curves gives the critical exponents $a$ and $b$ introduced in the text through equations (2) and (3).

or $b=\nu\left(d_{\min }-1\right)=0.17$. This estimate is an upper bound for the exponent $b$. The effective 
free extension could be smaller than the shorter path since there still exists some rigid clusters than cannot be deformed so as to adopt the conformation of the straight shortest path. However, the variation of the size of these rigid clusters is not critical in the vicinity of the usual percolation threshold; therefore we are led to conjecture that the free extension effectively scales as the upper bound given given above equation (4). We note that this theoretical estimate of $b$ is somewhat lower than the experimental value although within the error bar. This may be due to the fact that in the range of values of $p$ considered in figure $3 \mathrm{~b}$, the variation of the size of the rigid clusters is large and thus contribute to an apparent increase of $\lambda(q)$ with q.

The other critical behavior, in the vicinity of the $q_{\mathrm{r}}$, is much more difficult to handle. The reason for this is the non-local character of central-force percolation. In addition to this, we are dealing here with a property which is nonlinear, since it is the change of geometry of the original lattice which provides the constraints which make it rigid. However, we can suggest the following approximative picture : the lattice for $q$ close but larger than $q_{\mathrm{r}}$ consists in a set of rigid clusters, of typical size $\xi_{-}^{\prime}$ this correlation length is distinct from the previously introduced $\xi$. The length, $\xi^{\prime}$, diverges at the rigidity threshold with a critical index $\nu^{\prime}$ such that $\xi^{\prime} \propto\left(q-q_{\mathrm{r}}\right)^{-\nu^{\prime}} . \nu^{\prime}$ is characteristic of centralforce percolation. The only numerical determination of this exponent in given in reference [6] : $\nu^{\prime}=1.05 \pm 0.1$. Some other numerical studies [7] suggest on the contrary that central-force and usual percolation belong to the same universa. lity class, in which case $\nu^{\prime}=\nu=4 / 3$ would result. The rigid clusters are connected together by bonds which may move freely. Therefore, each cluster can be moved with respect to the other ones by an amount that is of the order of one bond length. The resulting overall extension of the lattice is thus of the order of $\left(L / \xi^{\prime}\right)$ or $\lambda(q) \propto L\left(q-q_{\mathrm{r}}\right)^{\nu^{\prime}}$, giving $a=\nu^{\prime}$. This estimate of $a$ is consistent with the experimental data (0.9).

The free extension of the lattice can be put in correspondence with a physical property of the "dual" central-force percolation. Suppose that in the lattice we replace all elastic bonds by infinitely rigid ones (fraction $1-q$ ), and all missing bonds by elastic ones (fraction $q$ ), we have a problem of "random reinforcement" $[7,10]$. The compliance, $C$, (defined as the inverse of the elastic modulus) of the lattice is zero for $q$ less than $q_{\mathrm{r}}$. It then increases continuously for $q$ up to 1 . Nothing appears to be critical in the neighborhood of $q_{\mathrm{c}}$. When a displacement $d$ is imposed onto the lattice, the tangent compliance of the lattice decreases to 0 when $d$ goes from zero to $\lambda(q)$ (with say $q_{\mathrm{r}}<q<q_{\mathrm{c}}$ ). Once again, we stress that the origin of this non-linear behavior is the existence of large deformations.

A natural question arises from the above considerations : what is the behavior of the compliance and of the elastic modulus, for the dual and for the original problem in the neighborhood of the critical line $\lambda(q)$ ? The only known answer is in the immediate vicinity of the $q_{\mathrm{r}}$, where the central-force percolation case is recovered, leading to the critical behavior of :

$Y$ in the original problem

$$
Y \propto\left(q_{\mathrm{r}}-q\right)^{f}
$$

and $C$ in the dual problem

$$
C \propto\left(q-q_{\mathrm{r}}\right)^{g}
$$

with $f / \nu^{\prime}=3$ and $g / \nu^{\prime}=0.97[7]$. More generally, for a given displacement $d$, there exists a value of $q=q^{*}$ such that $\lambda\left(q^{*}\right)=d$. In the vicinity of $q^{*}$ we expect $Y \propto\left(q^{*}-q\right)^{f(d)}$ for the original problem and $C \propto\left(q-q^{*}\right)^{g(d)}$ for the dual for a displacement of $d$ kept fixed. In addition, these transport exponents are to be complemented with an infinite series of exponents which describes the behavior of all moments of the force distribution in both problems (multifractal spectrum). Among those are two geometric exponents which give the fractal dimension of the force-carrying subset of bonds in the original problem. We know that, for $d=0$ or $q=q_{\mathrm{r}}$, we again find the usual backbone of central-force percolation, (with a fractal dimension of 1.93 as obtained in reference [6], or 1.65 from reference [7]). For $d$ tending to infinity, or $q=q_{\mathrm{c}}$, we have argued that this backbone is reduced to a set of shortest paths connecting finite rigid clusters whose size does not change critically around $q=q_{\mathrm{c}}$, thus leading to a fractal dimension of $d_{\min }=1.130$. Let us note that, in the latter case, the fractal dimension should be defined with respect to the undeformed geometry.

We now suggest the following picture for the size effects occuring in the interval $q_{\mathrm{r}}<q<$ 
$q_{\mathrm{c}}$. For $q^{*}(d)$, there exists a finite correlation length $\xi^{\prime}\left(q^{*}(d)\right)$ which gives the typical size of the rigid blobs. The properties of lattices of size $L<\xi^{\prime}$ should be exactly those of the centralforce percolation problem. Above this scale, we propose to renormalize the lattice by defining new sites as the rigid blobs, the connections between them becoming links. This "super-lattice" is a random structure of the percolation type with a lattice constant $\xi^{\prime}$; however, it is not at its threshold unless $q=q_{\mathrm{c}}$. Therefore, on the renormalized lattice we can define a new correlation length, $\xi^{\prime \prime}$, expressed in units of $\xi^{\prime}$, which will diverge for $q=q_{\mathrm{c}}$ as

$$
\xi^{\prime \prime} \propto\left(\xi^{\prime} \cdot\left(q-q_{\mathrm{c}}\right)^{-\nu}\right) \propto\left(q-q_{\mathrm{c}}\right)^{-\nu}
$$

with the usual $\nu$ exponent of percolation, since $\xi^{\prime}$ has a smooth variation close to $q_{\mathrm{c}}$. The evolution of the different length scales appearing in this picture has been schematically represented in figure 4. $\xi^{\prime \prime}$ diverges at $q=q_{\mathrm{r}}$ since this length scale is constructed with a lattice constant $\xi^{\prime}$ which itself diverges, however, the ratio $\xi^{\prime \prime} / \xi^{\prime}$ is of the order of 1 around $q_{\mathrm{r}}$. For a lattice of size $L$ such that $\xi^{\prime}<L<\xi^{\prime \prime}$ (labelled B in Fig. 4), one expects to see the usual percolation type properties (such as the one mentioned previously for the shortest path).

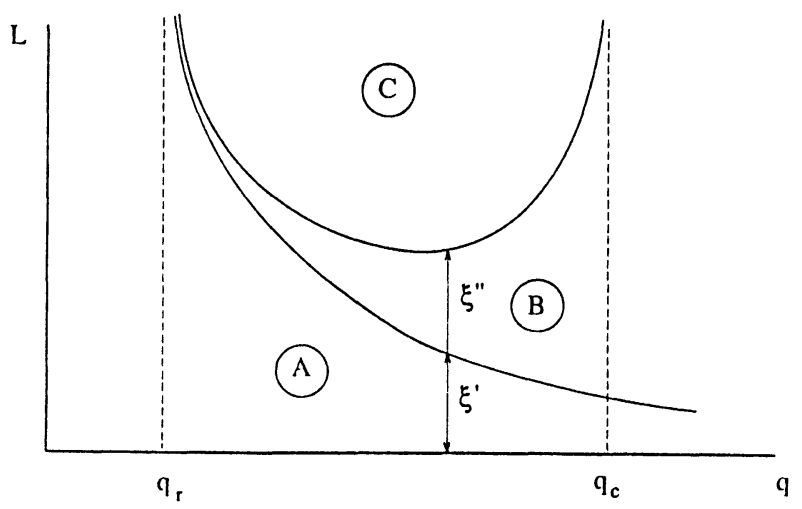

Fig. 4.- Schematic picture of the different length scales appearing for the case $q_{\mathbf{r}}<q<q_{\mathrm{c} . \ldots} \mathrm{A}$ : centralforce percolation regime $B$ : usual percolation regime _C : small-disorder regime.

The last regime ( $\mathrm{C}$ in Fig. 4) is different from both previous cases : for $L>\xi^{\prime \prime}$, the lattice structure is homogeneous; however, the length of the "super-links" fluctuates and we expect to see a "small-disorder regime" also encountered in other problems such as in packing of granular materials. With an infinitesimal force applied, a single path will be stretched. Progressively, as the force increases, the structure of the backbone will get richer and richer, and thus for instance, the elastic modulus will increase.

Finally, let us mention two experimentally important phenomenon which have not been taken into account in our study :

- steric hindrance effects which however will not modify the scaling properties obtained;

- in-plane buckling which might be of importance mainly before the rigidity threshold is reached, and that may change both the threshold and the critical exponents observed close to $q_{\mathrm{r}}$.

Chakrabarti et al. [11] have studied the elasticity of a solid made of atoms interacting via a Lennard-Jones potential (thus central-force but not linear) or not interacting at all with a certain probability and have also observed numerically the occurence of a finite free displacement if the fraction of non-interacting atoms was large. However, no quantitative results were extracted from their study about the quantities mentioned above.

Tang and Thorpe [12] have recently considered the problem of the evolution of the elastic modulus above $\lambda(q)$, in a so-called "stretched tennis-racket" problem and they provide a large amount of numerical results as well as a meanfield theory for it. The problem they addresses is close to the one we considered here. Although the qualitative feature of their results and ours do agree, a direct quantitative comparison is however difficult due to the difference of boundary conditions considered and to the fact that they did not investigate any critical aspect of the problem.

Finally, the results we have obtained experimentally and the questions they have raised certainly necessitate further investigations and application to more realistic materials where similar effects are expected to occur such as stretched unwowen textiles, or compressed piling of grains where the situation is somewhat symmetric to what we have described.

\section{Acknowledgments}

We would like to thank D. Sornette and C. Vanneste for useful discussions. 


\section{References}

[1] Kantor, Y. and Webman, I., Phys. Rev. Lett. 52 (1984) 1891.

[2] ZabolitzKY, J.G., Bergman, D.J. and Stauffer, D., J. Stat. Phys. 44 (1986) 211.

[3] Roux, S., J. Phys. A 19 (1986) L351.

[4] Benguigui, L., Phys. Rev. Lett. 53 (1984) 2028.

DEPTUCK, D., HARRISON, J.P. and ZaWADSKI, P., Phys. Rev. Lett. 54 (1985) 913.

[5] Feng, S., and Sen, P.N., Phys. Rev. Lett. 52 (1984) 216.

[6] LemieuX, M.A., BREton, P. and Tremblay, A.M.S., J. Phys. Lett. 46 (1985) L1;

DAY, A.R., TREMblay, R.R. and
Tremblay, A.M.S., Phys. Rev. Lett. 56 (1986) 2501.

[7] Roux, S. and HANSEN, A., Europhys. Lett. to appear;

HANSEN, A. and RouX, S., preprint.

[8] Feng, S., Thorpe, M.F. and GarboczI, E., Phys. Rev. B 31 (1985) 276.

[9] HerrmanN, H.J. and STANLEy, H.E., preprint;

Havlin, S. and Ben Avraham, D., $A d v$. Phys. 63 (1987) 695.

[10] Sahimi, M. and Goddard, J., Phys. Rev. $B 32$ (1985) 1869.

[11] Chakrabarti, B.K., Chowdhury, D. and Stauffer, D., Z. Phys. B 62 (1986) 343.

[12] TANG, W. and THORPE, M.F., preprint. 